\title{
Generation of subpicosecond $x$-ray pulses in storage rings
}

\author{
Alexander A. Zholents \\ Lawrence Berkeley National Laboratory \\ University of California, \\ Berkeley, California 94720
}

June 2007

\begin{abstract}
Disclaimer
This document was prepared as an account of work sponsored by the United States Government. While this document is believed to contain correct information, neither the United States Government nor any agency thereof, nor The Regents of the University of California, nor any of their employees, makes any warranty, express or implied, or assumes any legal responsibility for the accuracy, completeness, or usefulness of any information, apparatus, product, or process disclosed, or represents that its use would not infringe privately owned rights. Reference herein to any specific commercial product, process, or service by its trade name, trademark, manufacturer, or otherwise, does not necessarily constitute or imply its endorsement, recommendation, or favoring by the United States Government or any agency thereof, or The Regents of the University of California. The views and opinions of authors expressed herein do not necessarily state or reflect those of the United States Government or any agency thereof, or The Regents of the University of California.

Ernest Orlando Lawrence Berkeley National Laboratory is an equal opportunity employer.
\end{abstract}




\title{
GENERATION OF SUBPICOSECOND X-RAY PULSES IN STORAGE RINGS*
}

\author{
A.A. Zholents, LBNL, CA 94720, U.S.A.
}

\section{Abstract}

Supicosecond x-ray pulses are routinely produced at ALS, BESSY and SLS with "slicing" technique and used in pump-probe experiments with controlled delay between laser pump pulses and x-ray probe pulses. New development aiming for a production of a subpicosecond $\mathrm{x}$-ray pulses using rf orbit deflection technique is under way at APS. Both techniques will be reviewed here.

\section{INTRODUCTION}

Typical storage ring-based light source operates with electron bunches that are few tens of picosecond in length [1]. In a special mode of operation using low intensity bunches and significantly reduced momentum compaction factor one can obtain shorter bunches with the length of few ps [2]. Usually duration of the x-ray pulses emitted by these electron bunches is the same as duration of the electron bunch. However, subpicosecond (sub-ps) x-ray pulses can be obtained using either the radiation of a short slice of the electron bunch [3-7] or by compression of the x-ray pulses $[8,9]$. Methods to do it were developed during the past decade and will be briefly reviewed here.

\section{SLICING METHOD}

Sub-ps laser pulse can be used to create sub-ps time structure on a long electron bunch via energy modulation and subsequent spatial separation of an ultrashort slice of the bunch. Sub-ps synchrotron pulses (with a spectral range extending from infrared to $\mathrm{x}$-ray wavelengths) are then generated from the ultrashort electron slice. Figure 1 shows a schematic illustration of the energy modulation which is accomplished by co-propagating a femtosecond

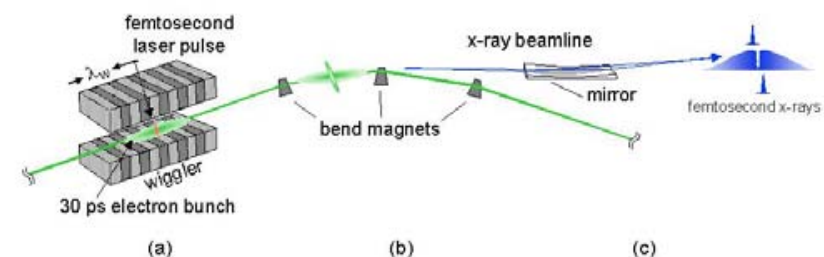

Figure 1. Schematic of the laser slicing method for generating sub-ps synchrotron pulses.

laser pulse with the stored electron bunch through a wiggler. The high electric field of the sub-ps laser pulse $\left(\sim 10^{9} \mathrm{~V} / \mathrm{m}\right)$ produces an energy modulation of electrons as they traverse the wiggler (electrons are accelerated or decelerated depending on the optical phase, $\phi$, seen by each electron at the entrance of the wiggler). The optimal interaction occurs when the central wavelength of the spontaneous emission from an electron passing through the wiggler, $\lambda_{\mathrm{s}}=\lambda_{\mathrm{w}}\left(1+\mathrm{K}^{2} / 2\right) / 2 \gamma^{2}$, where $\lambda_{\mathrm{w}}$ is the wiggler period, $\gamma$ is the Lorentz factor, and the deflection parameter $\mathrm{K}=\mathrm{eB}_{0} \lambda_{\mathrm{w}} / 2 \pi \mathrm{mc}$ (where $\mathrm{B}_{\mathrm{o}}$ is the peak magnetic field of the wiggler, $\mathrm{e}$ is the electron charge, $\mathrm{m}$ is the electron mass, and c is the speed of light), is close to the laser wavelength $\lambda$. In addition, the laser beam waist $w_{0}$ (assumed to be in the center of the wiggler) must exceed the horizontal and vertical electron beam sizes. Under these conditions, the electron energy modulation $\Delta E$ is given by [10]:

$$
\begin{aligned}
\Delta E=m c^{2} \sqrt{\frac{P_{L}}{P_{0}} 16 \pi N \frac{K^{2}}{2+K^{2}}}\{J J\} f\left(q, v, \hat{\sigma}_{\tau}\right) \cos (k s+\varphi) \\
f\left(q, v, \hat{\sigma}_{\tau}\right)=\sqrt{q} \int_{-0.5}^{0.5} d \bar{z}\left[\frac{\cos \left(2 \pi v \bar{z}-\tan ^{-1}(q \bar{z})\right)}{\sqrt{1+(q \bar{z})^{2}}}\right. \\
\left.\exp \left(-\left(\frac{\bar{z}}{2 \hat{\sigma}_{\tau}}-\frac{s}{2 c \sigma_{\tau}}\right)^{2}\right) \exp \left(-\frac{x^{2}+y^{2}}{w_{0}^{2}\left(1+(q \hat{z})^{2}\right)}\right)\right]
\end{aligned}
$$

where $P_{L}$ is the peak laser power, $P_{0}=I_{A} m c^{2} / e$ and $I_{A}$ is Alfvèn current, $k=2 \pi / \lambda$ is the wave vector, $\sigma_{\tau}$ is the rms length of the laser pulse intensity, $L_{w}$ is the length of the wiggler with $N$ periods, $\hat{\sigma}_{\tau}=\sigma_{\tau} / \tau_{0}$ and $\tau_{0}=2 \pi N / k c, \hat{z}=c t / L_{w}, v=N 2 \delta \gamma / \gamma, \delta \gamma / \gamma$ is the energy offset from the FEL resonance $\lambda_{s}=\lambda$, $q=L_{w} / z_{0}$, and $z_{0}=k w_{0}^{2} / 2$ is the Rayleigh length, $x, y$ are the electron coordinates at the entrance of the wiggler, $s$ is the electron coordinate within the electron bunch, $\quad\{J J\}=J_{0}(\xi / 2)-J_{1}(\xi / 2) \quad$ where $\xi=K^{2} /\left(2+K^{2}\right)$ and $J_{0}$ and $J_{1}$ are the first and the second order Bessel functions of the first kind.

Table 1 shows peak relative amplitude of energy modulation $\Delta \mathrm{E} / \mathrm{E}_{\mathrm{b}}$ (where $\mathrm{E}_{\mathrm{b}}$ is the electron beam energy) at various facilities calculated using (1) for a non distorted laser beam $\left(\mathrm{M}^{2}=1\right)$ with $\sigma_{\tau}=21 \mathrm{fs}, \lambda=800 \mathrm{~nm}$, and a pulse energy $1 \mathrm{~mJ}$.

Table 1: Peak amplitude of energy modulation

\begin{tabular}{|c|c|c|c|}
\hline Facility & $\mathbf{E}_{\mathbf{b}}(\mathbf{G e V})$ & $\mathbf{N}$ & $\Delta \mathbf{E} / \mathbf{E}_{\mathbf{b}}(\mathbf{\%})$ \\
\hline ALS & 1.9 & 29 & 1.3 \\
\hline BESSY & 1.7 & 10 & 1.0 \\
\hline SLS & 2.4 & 17 & 0.9 \\
\hline
\end{tabular}

The amplitude of the energy modulation versus laser pulse energy measured in BESSY is shown in Figure 2 in comparison with theoretical prediction. One can see that approximately one half of the laser pulse was used to reproduce the experimental results [6]. Similar observation was reported in [5] for ALS. No plausible 
explanation for this discrepancy had been found so far except the assumptions of a possible distortion in the laser wave fronts across the electron beam and that the laser pulse delivered to the middle of the wiggler is somehow longer than presumed and carries less energy.

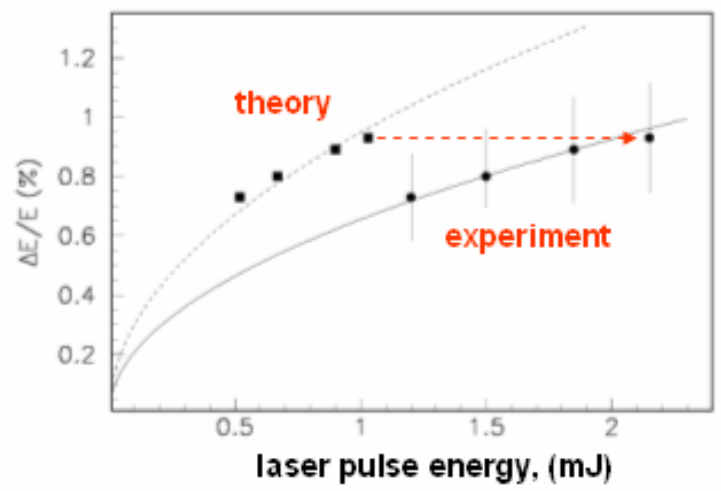

Figure 2. Energy modulation amplitude versus laser pulse energy [6].

After the wiggler, the electron bunch passes a set of dispersive elements that separate electrons with large amplitude of energy modulation. Then the radiation of these electrons is selected in the x-ray beamline. One can use either coordinate separation and do a selection in the image plane $[4,11]$ (see Figure 1) or angular separation and do a selection right at the exit of the source $[6,7]$ (see Figure 3).

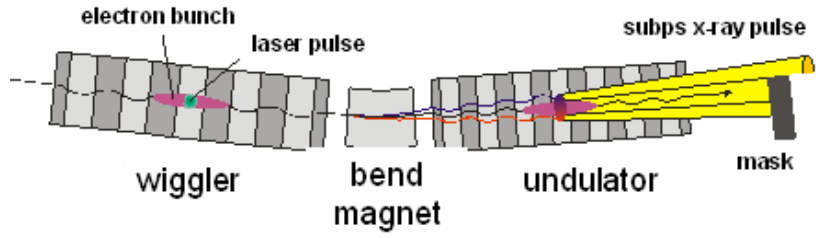

Figure 3. A schematic of angular separation of the sub-ps xray pulses emitted in the undulator with the mask blocking the radiation of core electrons at the undulator exit [6].

Because no x-ray mirrors is placed in front of the mask in the second case, it is much easier to obtain a large signal to background ratio there than in the first case where background is affected by non-specular reflections in the $\mathrm{x}$-ray optics of the emission of core electrons (see Figure 4).

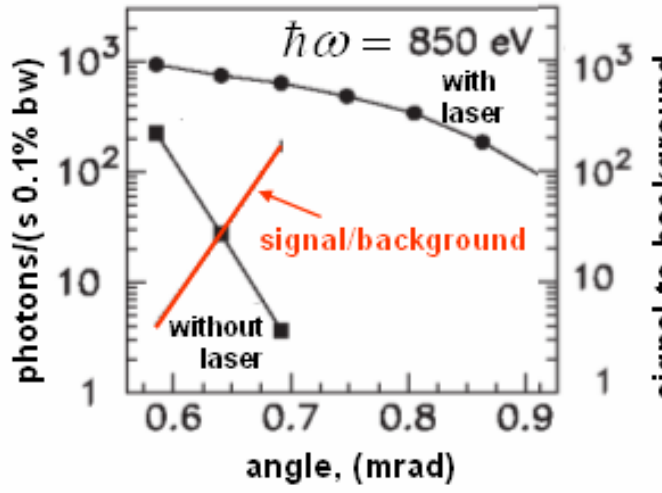

Figure 4 . Detected photon rate per $0.1 \%$ bandwidth and signalto-background ratio versus cutoff angle [6].
The time-of-flight properties of the storage ring cause electrons with $\Delta \mathrm{E}<0$ to follow a shorter path and therefore shift toward the head of the bunch, while electrons with $\Delta \mathrm{E}>0$ follow a longer path and shift toward the tail of the bunch. In result, the electron density distribution in the vicinity of the energy modulated slice undergoes continuous evolution as the electron bunch propagates along the storage ring, first showing a formation of a dip then its disappearance. Similarly, dispersion properties of the lattice cause a horizontal displacement $\Delta \mathrm{x}$ of nonequilibrium electrons. Figure 5 shows $2 \mathrm{D}$ electron density distribution calculated in the vicinity of the modulated slice after electron bunch propagation 1.5 sector of the ring from the wiggler and Figure 6 shows a dip in 1D electron density distribution formed at this location.

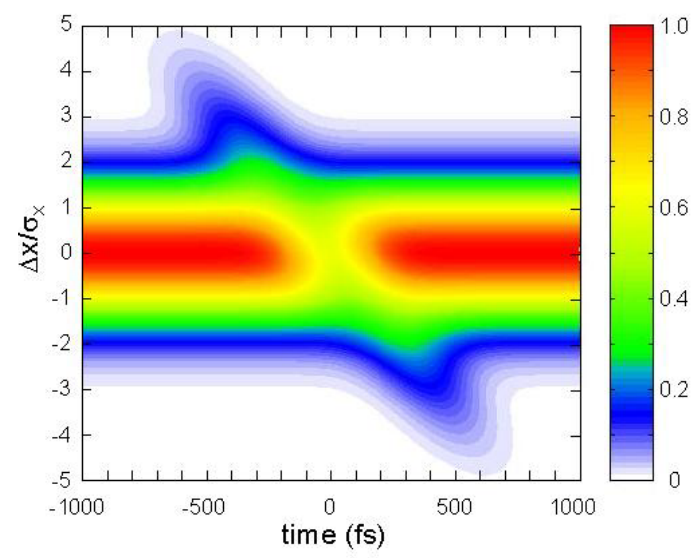

Figure 5. A calculation of the 2D electron density distribution (as a function of time and horizontal displacement normalized on the rms size) after electron bunch propagation 1.5 arc sectors at the ALS from the wiggler to the bend magnet [4].

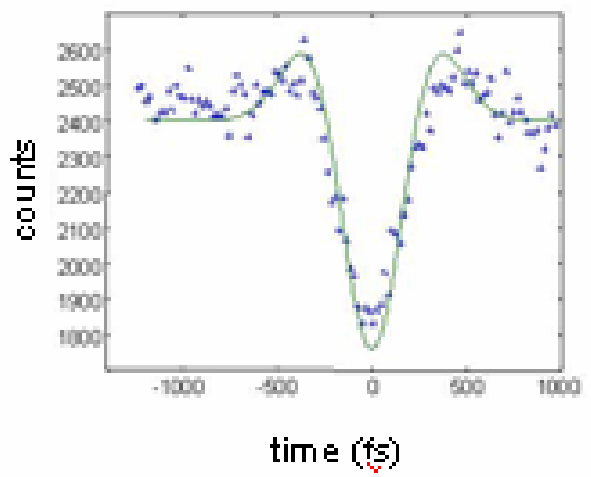

Figure 6. A dip in the 1D electron density distribution measured at the ALS at a bend magnet located 1.5 arc sectors from the wiggler [4].

The duration of the x-ray pulse produced by slicing is determined by duration of the laser pulse, slippage of the electrons with respect to the light in the wiggler and by the time-of-flight dispersion on a path from the wiggler to the radiator. This dispersion is inevitable since lattice dispersion is used for a selection of the femtosecond radiation and, therefore, the lattice between the aforementioned points is non achromatic and non isochronous. The above mentioned partition contributions 
and a total x-ray pulse duration calculated for various facilities are given in Table 2.

Table 2: Pulse stretching partition contributions and the total duration of the x-ray pulse (fwhm in femtoseconds).

\begin{tabular}{|c|c|c|c|c|}
\hline Facility & $\begin{array}{c}\text { Laser } \\
\text { pulse }^{\mathbf{1}}\end{array}$ & Slippage & $\begin{array}{c}\text { Lattice } \\
\text { dispersion }\end{array}$ & Total \\
\hline ALS & 100 & 70 & 180 & 200 \\
\hline BESSY & 70 & 26 & 65 & 100 \\
\hline SLS & 70 & 45 & 125 & 150 \\
\hline
\end{tabular}

1) given as FWHM of the electric field

\section{Diagnostics}

Direct measurement of sub-ps x-ray pulses is difficult and reliable methods have yet to be developed, but one can do it indirectly. Various diagnostics technique had been used. Since time structure of the electron bunch spontaneous emission is invariant over the entire spectrum of the spontaneous emission, one can use visible synchrotron radiation ( $\sim 2-\mathrm{eV}$ photon energy) from a bend magnet instead of the x-rays and cross-correlate it with the light from a sub-ps laser pulse. Following this idea a visible light from ALS bend magnet was imaged onto a nonlinear optical crystal, along with a delayed 50 -fs pulse from the laser system. Photons at the sum frequency ( $\sim 3.5-\mathrm{eV}$ photon energy) were counted as a function of delay between the modulating laser pulse (propagating through the wiggler) and the laser pulse used for crosscorrelation. An adjustable knife edge located in the beamline at an intermediate image plane of the synchrotron radiation (before the nonlinear optical crystal) was placed at the $3 \sigma_{x}$ position to reveal the radiation pulse shown in Figure 7.

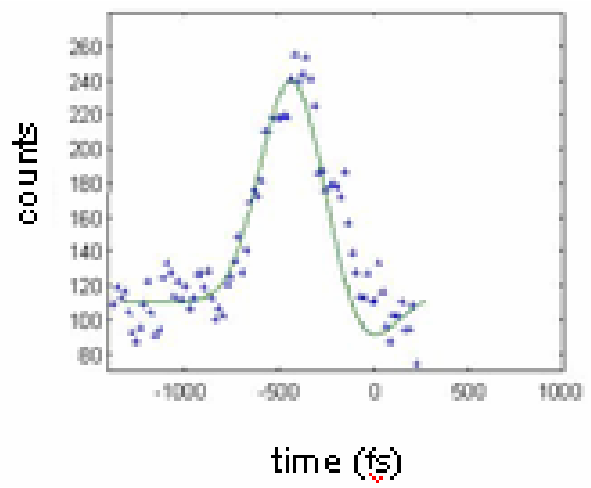

Figure 7. A pulse of synchrotron radiation from the bend magnet measured with knife edge at the $3 \sigma_{\mathrm{x}}$ position [4].

The electron density modulation shown in Figure 6 gives rise to a coherent synchrotron radiation [5]. This radiation was detected at ALS [12], BESSY [13] and SLS [14]. The spectrum of the signal $|F(\omega)|$ measured with liquid He-cooled $\mathrm{Si}$ bolometer and Martin-Puplett spectrometer [13] is shown in Figure 8. Since $F(\omega)$ is a Fourier transform of the $1 \mathrm{D}$ electron density distribution and because the shape of the dip in the electron density distribution can be accurately modeled with two Gaussians functions, one can reconstruct the original distribution from the measured spectrum using either Kramers-Kronig relation [15] or iteratively. The result is shown in Figure 9 along with macroparticle simulation.

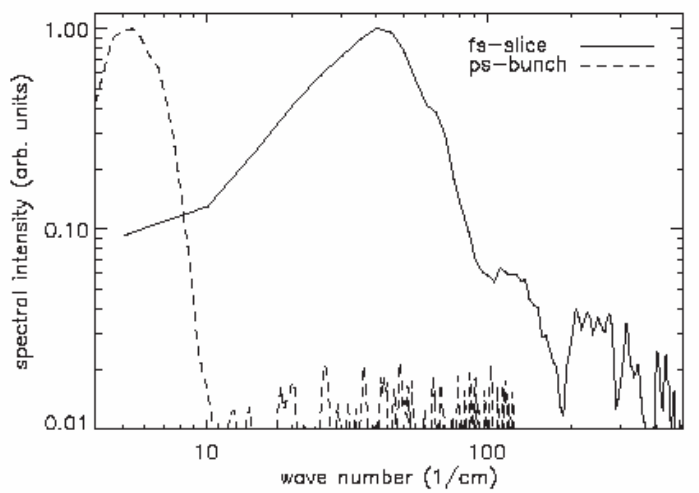

Figure 8 . The spectrum of the coherent synchrotron radiation produced by the electron density modulation (solid line) and the spectrum of the $1.2 \mathrm{ps}$ (rms) electron bunch coherent radiation obtained in the operation with a low momentum compaction factor (dashed line) [13].
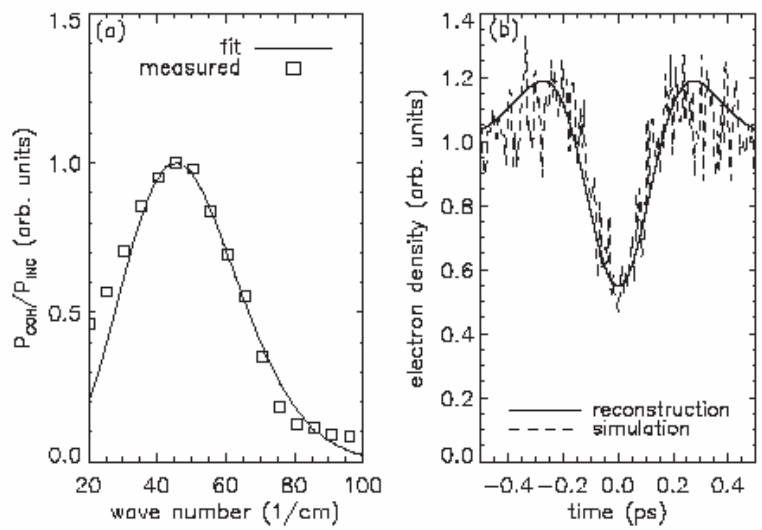

Figure 9. The spectrum (a) and the reconstructed electron density distribution (b). Dashed line in (b) shows the result of the macroparticle simulation [13].

It was found at ALS, BESSY and SLS that coherent synchrotron radiation provides an excellent $\mathrm{THz}$ signal clearly visible on many turns after electron bunch interaction with the laser pulse. Therefore, it is now routinely used for an initial optimization of the laserelectron beam interaction and for a feedback control of spatial and timing drifts between laser and electron beams during data logging in experiments with sub-ps x-ray pulses. It was also empirically found at BESSY that the ratio of $\mathrm{THz}$ pulse intensity on the first and the second turn is extremely sensitive to the initial energy modulation of electrons [13].

\section{Toward subfemtosecond $x$-ray pulses}

A modification to the slicing source of sub-ps $x$-ray pulses was recently considered in [16]. It was proposed to use interaction of the electrons with the laser pulse in the 
$\mathrm{TEM}_{01}$ Hermite-Gaussian mode. In this case there is no need in lattice dispersion as interaction itself produces angular modulation of electrons that can be used for a separation of sub-ps x-ray pulses. This eliminates a need in bending magnets between wiggler and radiator formally responsible for stretching of the x-ray pulse. The coordinate separation in the vertical plane is preferred in this scheme as it requires lesser laser pulse energy. Figure 10 shows possible implementation where we use the undulator in a location with a $90^{\circ}$ betatron phase advance from the wiggler.

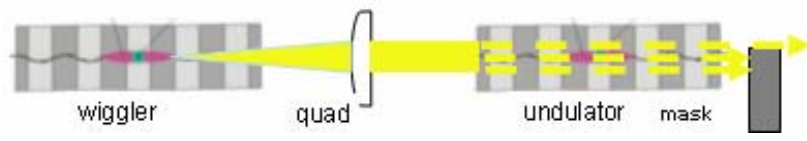

Figure 10. A schematic of a slicing technique utilizing the laser pulse in the $\mathrm{TEM}_{01}$ Hermite-Gaussian mode.

Thus, with isochronous lattice between wiggler and undulator, generation of sub-fs x-ray pulses becomes feasible in principle. Following the idea initially put forward for free electron lasers [17], one can employ a wiggler with just one period and a few-cycle laser pulse with a carrier-envelop phase stabilization to produce angular modulation shown in Figure 11. In principle, this modulation allows a selection of the sub-femtosecond $\mathrm{x}$ ray pulse if background radiation is solely defined by $\mathrm{x}$ ray diffraction. We presume that non Gaussian tails in the electron distribution can be cleaned by kicking the electron bunch towards the scraper right before the interaction with the laser pulse.

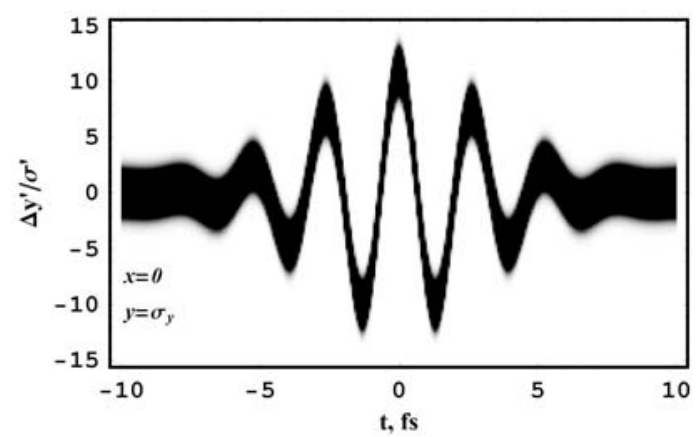

Figure 11. Density plot for a fragment of the electron bunch showing angular modulation of the electrons with coordinates $x=0, y=\sigma_{y}$ obtained in the interaction with a $5 \mathrm{~mJ}$ energy, 5

fs FWHM long laser pulse in $\mathrm{TEM}_{01}$ mode in a wiggler magnet with one period.

\section{RF ORBIT DEFLECTION}

A success in generation of sub-ps x-ray pulses largely depends on a condition when radiation of a slice of the electron bunch is clearly separated from the radiation of all other electrons. Taking this idea further one can envision a situation where an entire electron bunch is seen as made out of a large number of separated slices producing the radiation that do not overlap. This what an rf orbit deflection technique proposed in [8] and independently in [9] does. Figure 12 provides an illustration to the idea.

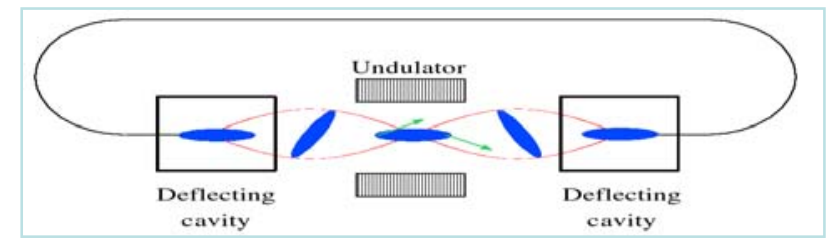

Figure 12. A schematic of an $\mathrm{rf}$ orbit deflection technique for generation of sub-ps x-ray pulses.

An electron bunch passing a deflecting cavity receives a time correlated kick such as head electrons are kicked up and tail electrons are kicked down. Then electrons follow betatron oscillations and appear in the undulator (located at a half of the betatron wave away from the cavity) on trajectories where the head electrons go down and the tail electron go up. Consequently, they produce an undulator radiation that goes down in the case of head electrons and up in the case of tail electrons. If an angle between head and tail radiation is larger than a combination of a diffraction limited angle of the undulator radiation $\sigma_{r^{\prime}}=\sqrt{\lambda_{x} / \pi L_{u}}$ (where $\lambda_{x}$ is the $\mathrm{x}$-ray wavelength and $L_{u}$ is the undulator length) and an rms angular spread of electrons $\sigma_{y^{\prime}}$ in undulator, then head and tail radiation can be treated independently as produced by two independent sources. In fact, what we really want is that this angle will be large enough that radiation produced by many more slices of the electron bunch between head and tail can be treated independently and be compressed into a single $\mathrm{x}$-ray pulse using asymmetrically cut crystal as shown in Figure 13.

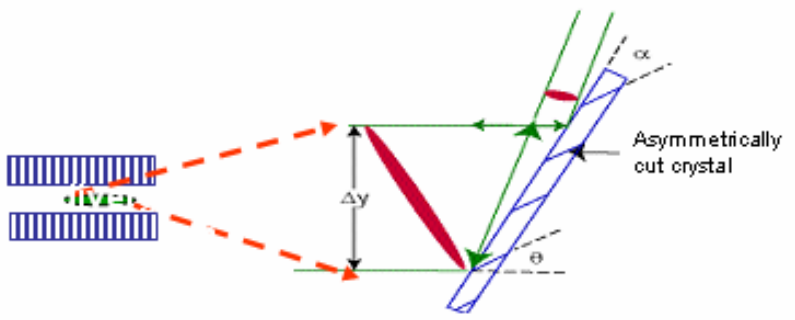

Figure 13. An illustration of an x-ray pulse compression using asymmetrically cut crystal [8].

One can estimate the length of the x-ray pulse obtained after compression using the following formula:

$$
\sigma_{x-r a y} \approx \frac{E_{b}}{e U} \frac{\lambda_{r f}}{2 \pi} \sqrt{\frac{\varepsilon_{y}}{\beta_{r f}}\left(1+\frac{\sigma_{r^{\prime}}^{2}}{\sigma_{y^{\prime}}^{2}}\right)}
$$

where $U$ is the integral of the electric field taken along the line with a maximum amplitude of the field, $\lambda_{r f}$ is the rf wavelength, $\varepsilon_{y}$ is vertical emittance, $\beta_{r f}$ is vertical beta function in the cavity.

Second cavity in Figure 12, located one betatron wavelength apart from the first cavity, is used to cancel 
the effect of first cavity. This is important in order to keep $\varepsilon_{y}$ small and to be able to achieve a smallest possible length of the x-ray pulse according to (2). However, feasibility studies of the rf deflection technique for APS [18] revealed a large blow-up of the vertical emittance [19]. It was then found that this was mainly caused by sextupole lenses used for chromaticity correction between cavities and a variant of chromaticity correction using a new set of sextupole gradients was found where emittance blow-up was at an acceptable level [20]. The blow-up of the vertical emittance and emittance oscillations that accompany it were eventually understood as mainly driven by coupling of horizontal and vertical emittances [21] (see, Figure 14).

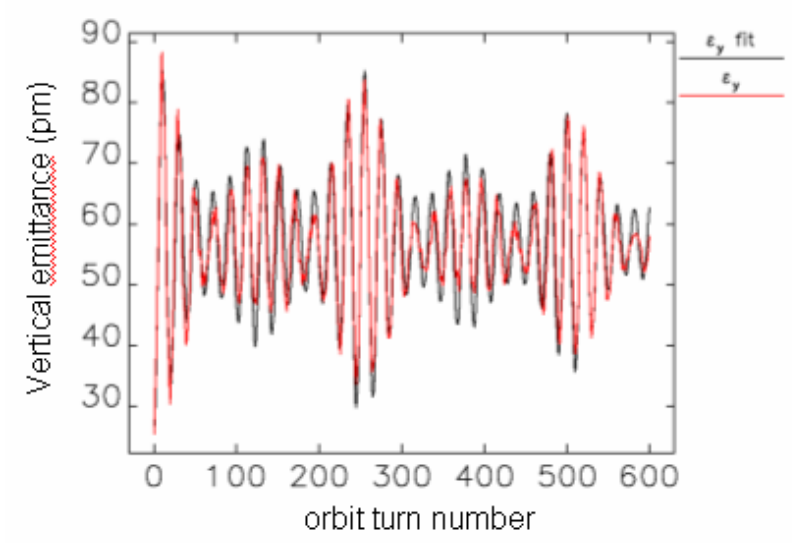

Figure 14. Transient emittance oscillations caused by coupling of the horizontal and vertical emittances during first several hundred orbit turns after switching on of the deflecting cavities. The red line shows simulations starting from initial emittance of $\sim 25 \mathrm{pm}$ and the black line shows a theoretical fit [21].

An interesting idea also involving tilting the electron bunch has been proposed and tested in [22]. The entire electron bunch was kicked vertically using a fast kicker and performed free betatron oscillations afterward. Over one half of a period of synchrotron oscillations the head and the tail electrons developed a measurable phase shift in oscillations because of the ring chromaticity. It was then possible to find a location where, say, head electrons went up and tail electrons went down, thus, producing a tilt of the electron bunch that can be estimated using the following formula [22]:

$$
\frac{d y}{d z} \approx \Delta y_{0}^{\prime} \beta_{y} \frac{4 \pi C_{y}}{L \alpha}
$$

where $z$ is the coordinate along the electron bunch, $\Delta y_{0}^{\prime}$ is the initial kick, $\beta_{y}$ is the average vertical betafunction, $C_{y}$ is the vertical chromaticity of the ring, $\alpha$ is the momentum compaction factor, and $L$ is the ring circumference.

\section{SUMMARY}

The "slicing" technique for generation of sub-ps x-ray pulses in a storage ring had been successfully implemented at ALS, BESSY and SLS. All three facilities produced experimental results, some already published $[23,24]$ and some in a process of publication $[25,26]$. The technique of the rf orbit deflection had been intensively studied for APS and soon will be tested there [27-29]. These facilities permit us to have a glimpse of a microworld with a sub-ps resolution that will eventually be scrutinized when $\mathrm{x}$-ray free electron lasers will come to life.

Acknowledgement. This work was supported by U.S. DoE Contract No: DE-AC02-05CH11231.

\section{REFERENCES}

[1] http://www.als.lbl.gov/als/synchrotron_sources.html.

[2] M. Abo-Bakr, J.Feikes, K. Holldack, G. Wüstefeld, H.-W. Hübers, Phys. Rev. Lett, 88, 254801 (2002).

[3] A.A. Zholents and M.S. Zolotorev, Phys. Rev. Lett, 76, 912 (1996).

[4] R.W. Schoenlein et al., Science, March 24, (2000).

[5] R.W. Schoenlein et al., Appl. Phys. B71, 1 (2000).

[6] S. Khan et al., PRL, 97, 074801 (2006).

[7] A. Streun et al., in Proc. 2006 Europ. Part. Acc. Conf., Edinburg, THPLS062 (2006).

[8] A. Zholents, P. Heimann, M. Zolotorev, J. Byrd, NIM A, 425, 385 (1999).

[9] M. Katoh, Japan. J. Appl. Phys, 38, L547(1999).

[10]A.A. Zholents, K. Holldack, in Proc. 2006 Free Electron Laser Conf., Berlin, (2006).

[11] C. Steier et al., in Proc. 2005 Part. Acc. Conf., Knoxville, 4096 (2005).

[12] J. Byrd et al. , Phys. Rev. Lett., 96, 164801 (2006).

[13] K. Holldack et al., Phys. Rev. Lett., 96, 054801 (2006).

[14] V. Schlott et al., in Proc. 2006 Europ. Part. Acc. Conf., Edinburg, TUPCH094 (2006).

[15]R. Lai and A.J. Sievers, Phys. Rev. E, 53, 6413 (1996).

[16] A.A. Zholents and M. S. Zolotorev, LBNL Preprint, LBNL-62750, May (2007), unpublished.

[17] A.A. Zholents and W.M. Fawley, Phys. Rev. Lett., 92, 22480 (2004).

[18]K. Harkay et al., in Proc. 2005 Part. Acc. Conf., Knoxville, 668 (2005).

[19] M. Borland, Phys. Rev. ST-AB, 8, 074001, (2005).

[20] M. Borland and V. Sajaev, in Proc. 2005 Part. Acc. Conf., Knoxville, 3888 (2005).

[21] V. Sajaev, A. A. Zholents, LBNL Preprint, LBNL62852, June (2007), unpublished.

[22] W. Guo et al., Phys. Rev. ST-AB, 10, 020701, (2007).

[23]A. Cavalleri et al., Phys. Rev. Lett., 95, 067405 (2005).

[24] A. Cavalleri et al., Nature, 442, 664 (2006).

[25] C. Stamm, private communication.

[26] G. Ingold et al., PSI Scientific Report 2006.

[27] V. Sajaev et al., to be published in Proc. SRI 2007, Baton Rouge, (2007).

[28] M. Borland, TUPMN091 (ID-2913), this conference. [29]B. Yang, TUPMN104 (ID-2813), this conference. 
